\title{
Pharmacodynamics of Isoflupredone Acetate in an Endotoxin-Induced Mastitis Model
}

\section{S. A. Wagner and M. D. Apley}

Department of Veterinary Diagnostic and Production Animal Medicine College of Veterinary Medicine

lowa State University, Ames 50011

\section{ABSTRACT}

The effect of intravenous administration of the steroidal drug isoflupredone acetate on lactating dairy cows with mastitis induced using gram-negative bacterial endotoxin was investigated. Cows were randomly assigned to one of four treatment groups: untreated controls, isoflupredone acetate only, mastitis only, and mastitis plus isoflupredone acetate. Isoflupredone acetate was given to treated groups at a dose of $20 \mathrm{mg}$ intravenously, once. Mastitic cows receiving treatment were given isoflupredone acetate after the development of clinical signs. When compared with untreated mastitic controls, cows with endotoxin-induced mastitis treated with isoflupredone acetate did not exhibit measurable differences in heart rate, rectal temperature, rumen motility, or changes in mammary gland surface area in the $14 \mathrm{~h}$ following the administration of intramammary endotoxin. Healthy cows treated with isoflupredone acetate had a higher heart rate over the $14 \mathrm{~h}$ after drug administration than did untreated healthy controls. When compared with untreated mastitic controls, cows treated with isoflupredone acetate did not exhibit statistically significant differences in milk production following endotoxin-induced mastitis. (Key words: mastitis, steroid, milk production)

Abbreviation key: LPS = lipopolysaccharide.

\section{INTRODUCTION}

Mastitis is a common affliction of modern dairy cows and a major cause of lost income in the dairy industry. Temporary or permanent decreases in milk production may result from inflammatory damage to the mammary gland caused by mastitis. Antimicrobial drugs can inhibit or destroy mastitis-causing bacteria, but antimicrobial drugs do not generally have any anti-inflamma-

Received July 11, 2002.

Accepted September 10, 2002.

Corresponding author: S. A. Wagner; e-mail: sawagner@ iastate.edu. tory activity, thus they do not act directly to limit inflammatory damage to the mammary gland (Nickerson et al., 1986).

Researchers in the past have investigated whether therapy of gram-negative endotoxic mastitis with steroidal or nonsteroidal anti-inflammatory drugs is beneficial. Typically, investigators have administered a variety of anti-inflammatory drugs concurrently with or shortly after the induction of mastitis using bacteria or purified bacterial endotoxin, but before the development of systemic clinical signs.

Unfortunately, in a clinical setting it is not possible to identify and treat mastitic cows before the development of clinical signs of mastitis. The goal of this project was to test the hypothesis that the steroidal drug isoflupredone acetate may limit milk production loss and reduce clinical signs in cows with endotoxin-induced mastitis when the drug is given parenterally after the development of clinical signs. In the United States, the steroidal drug isoflupredone acetate is labeled for the treatment of inflammatory conditions in dairy cows.

\section{MATERIALS AND METHODS}

This protocol was approved by the Committee on Animal Care at Iowa State University.

\section{Study Population}

Thirty-two Holstein cows from the Iowa State University Dairy in Ankeny, Iowa, were used in this study. They were selected based on the following criteria: 30 to $70 \mathrm{~d}$ into second or greater lactation, no clinical mastitis in the current lactation, no drug treatment in the previous $30 \mathrm{~d}$, and no abnormalities on physical examination.

\section{Group Assignment and Randomization Procedures}

Cows were randomly assigned to one of four treatment groups: untreated controls, drug administration only, mastitis only, and mastitis plus drug administra- 
tion. Randomization was achieved by assigning a twodigit random number from a random number list, read in sequence from a random starting point, to each treatment group on each day. Cows were then assigned to a treatment group by ranking each cow from lowest to highest farm identification number, then assigning them to the group with the randomly assigned number of the same rank. To minimize day effects on clinical outcomes, one cow from each of the four treatment groups was enrolled in the trial on each of eight trial days. Two of the 32 selected cows were removed from the study due to lameness on the day they were scheduled to participate, leaving a total of 30 cows enrolled in the study. Ultimately there were eight cows in each treated group and six cows in the control group, as both cows removed due to lameness had been assigned to the untreated control group. All clinical work for this project was conducted between September 2000 and April 2001.

\section{Housing and Husbandry}

On trial days, cows were kept in a small barn where they were accustomed to spending time while undergoing treatments and routine examinations such as pregnancy diagnosis. They had unlimited access to water and a TMR composed primarily of corn silage and dry hay, formulated for lactating dairy cows. Cows were caught in headlocks for the hourly recording of clinical observations but were allowed to roam freely in the barn at other times. The cows were milked at their normal 12-h interval.

\section{Induction of Mastitis}

Cows assigned to treatment groups with mastitis had a negative bacterial culture $72 \mathrm{~h}$ before the induction of mastitis and a negative California Mastitis Test in the treated quarter on the day of their participation study. The California Mastitis Test on the other three quarters had either negative or trace reaction immediately before the induction of mastitis.

Mastitis was induced in the left front quarter of selected cows unless there was a trace positive California Mastitis Test in that quarter on the day of the study, in which case the right front quarter was used. Mastitis was induced in selected quarters by intramammary administration of $0.1 \mathrm{mg}$ of purified lipopolysaccharide (LPS) from Escherichia coli B4:0111 (Sigma-Aldrich, St. Louis, MO) in 20-ml sterile isotonic saline solution.

Clinical mastitis was defined as measurable swelling of the affected mammary gland, defined as an increase above baseline measurements of mammary surface area, and a rise in rectal temperature of at least $1^{\circ} \mathrm{C}$ above the baseline measurement taken before the administration of LPS. Cows that did not meet these criteria for development of clinical mastitis following the infusion of LPS into a mammary gland were removed from the study.

Eighteen groups of cows were cultured in order to qualify the nine groups of cows enrolled in the study. One group of four cows was removed from the study on the day of participation because the cows in the groups given intramammary endotoxin did not develop clinical mastitis as defined in this study.

\section{Clinical Variables}

Clinical variables recorded hourly for all treatment groups included rectal temperature by electronic thermometer, heart rate by auscultation for $15 \mathrm{~s}$ with a stethoscope, and number of rumen contractions per 2 min by auscultation for 2 min with a stethoscope. Cows in groups with mastitis also had mammary skin surface area measured hourly. This was done using a modification of the technique of making two indelible marks on the udder skin and measuring the distance between the marks using calipers, as described by Bywater et al. (1988). In this study, four indelible marks were made on the udder skin using a rectangular plastic template measuring $100 \mathrm{~mm} \times 120 \mathrm{~mm}$, and the distance between the marks for each side of the rectangle and both diagonals were measured using a flexible plastic ruler. Total area of the rectangle at each time point was calculated as follows: The rectangular area being measured was divided by the two diagonals in to four triangles, and the area of each triangle was calculated based on the length of its three sides using Heron's Formula, which states that the area of a triangle with sides of lengths $\mathrm{a}, \mathrm{b}$, and $\mathrm{c}$ is equal to $\sqrt{s(s-a)(s-b)(s-c)}$, where $\mathrm{s}$ equals $\frac{a+b+c}{2}$. The areas of the four triangles were summed to total the overall rectangular surface area. This method allowed surface area measurements to accurately reflect expansion that occurred outward from the initial plane of measurement, in addition to expansion within the two dimensions of the created rectangle.

\section{Study Design}

One cow from each treatment group was enrolled in the study on each trial day, in order to minimize day effects on outcome variables.

Beginning immediately after morning milking, each cow was examined and had baseline clinical observation values recorded. Cows in groups with endotoxin-induced mastitis were prepared for udder surface area measurement by clipping the hair of the mammary 


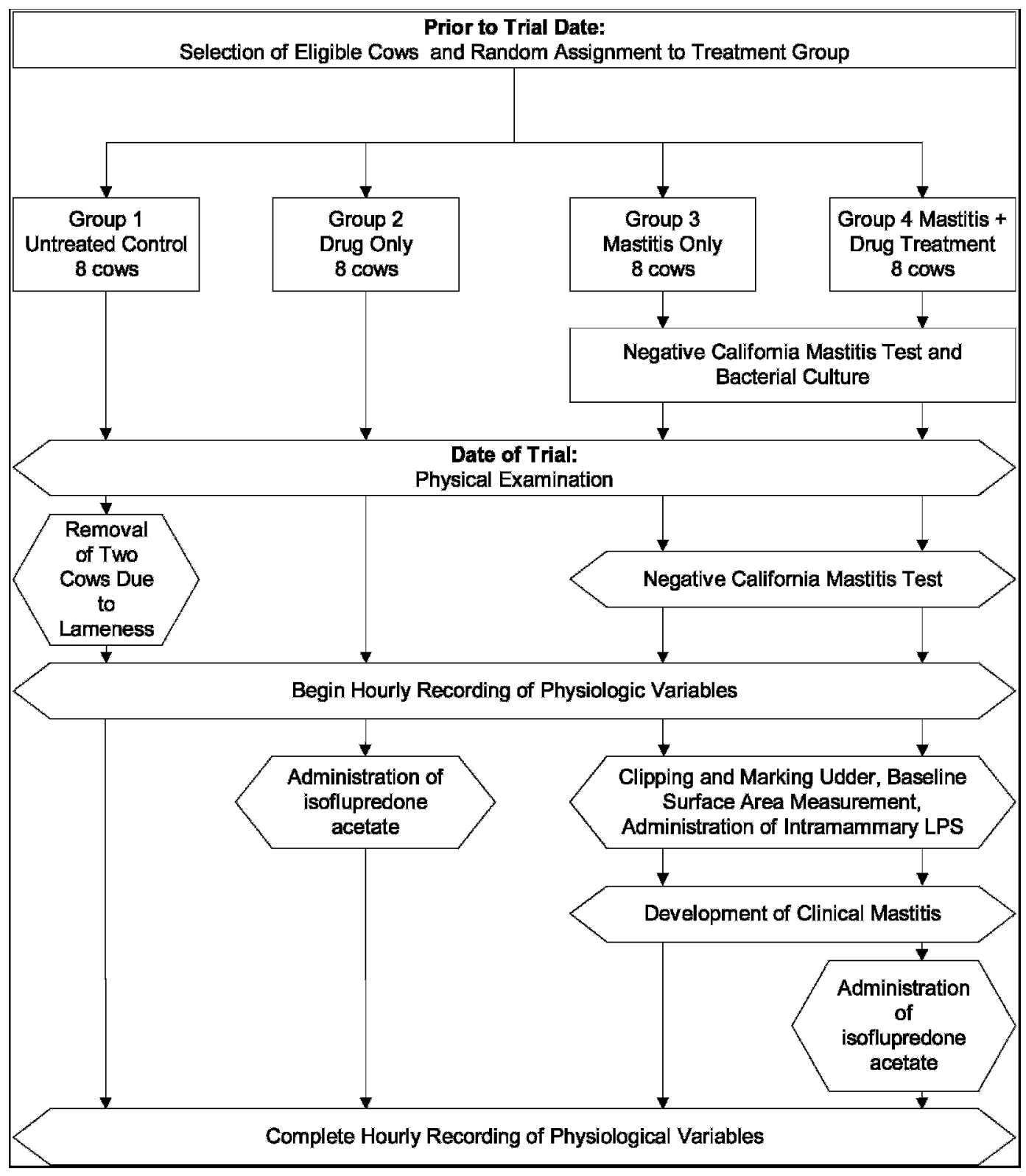

Figure 1. Flowchart of study procedures.

gland quarter to be treated and placing four black marks on that quarter using a plastic template and a permanent marker. The four experimental groups were then treated as follows (Figure 1):

Cows in the untreated control group had clinical variables recorded for 14 more hourly observations (15 total observations including baseline).

Cows in the group receiving only drug treatment were given $20 \mathrm{mg}$ of isoflupredone acetate (Predef $2 \mathrm{X}$, Pharmacia Animal Health, Kalamazoo, MI) one time by jugular venipuncture soon after the recording of baseline observations. Clinical variables were recorded for 14 more hourly observations. Cows in this group also had nine blood samples taken by jugular venipuncture over the next $24 \mathrm{~h}$ for a concurrent pharmacokinetic study.

Cows in the group with endotoxin-induced mastitis but no drug treatment had LPS infused into one quarter shortly after the recording of baseline variables. Mammary surface area measurements and other clinical variables were recorded for 14 more hourly observations.

Cows in the group receiving drug treatment following the development of clinical mastitis had LPS infused into one quarter shortly after the recording of baseline 
variables. Cows that subsequently developed clinical signs of mastitis as defined above received $20 \mathrm{mg}$ of isoflupredone acetate one time by jugular venipuncture as soon as possible after the hourly observation at which they were noted to have developed clinical mastitis. Observations were continued for at least $12 \mathrm{~h}$ following the establishment of clinical mastitis. This group also had nine blood samples collected by jugular venipuncture over a 24-h period for a concurrent pharmacokinetic study.

\section{Milk Production}

Milk weight data used in this study were collected electronically. Each cow in the study wore a computer chip bearing her farm identification number on a neck strap. This identification number was read by a scanner as each cow entered the milking parlor and was used to record the cow's milk production at each milking. Cows enrolled in this study had twice-daily milk weights recorded for the week before and $3 \mathrm{wk}$ after the day of participation in the study.

\section{Statistics}

All statistical analyses for this study were performed using the JMP statistical software program (SAS Institute, Cary, NC). The study design provided a power of greater than 0.80 to detect a 1-day difference of 10 $\mathrm{lb}$ in daily milk weight between groups. Analysis of baseline clinical variables was performed using analysis of variance. Subsequent clinical variables and 4day baseline milk production data were analyzed using multivariate analysis of variance, which accounts for repeated measurements over time. Statistical significance was designated a priori as a $P$ value less than or equal to 0.05 . Baseline clinical observations were considered covariates for statistical analysis of clinical variables following treatment, in order to compensate for variation in mean baseline measurements between groups.

Initial analysis of milk production during the recovery period using multivariate analysis of variance for repeated measures revealed an interaction between time and treatment. Further statistical analysis was therefore done using analysis of variance separately for each day of the recovery period. Dunnett's method was used to correct the tendency toward type 1 error when comparing treatment groups individually to the control group.

Due to failure of the scanner at the milking parlor entrance to read the cow's identification number, milk weight data were not recorded for every cow at each time point used in the data analysis. Missing milk

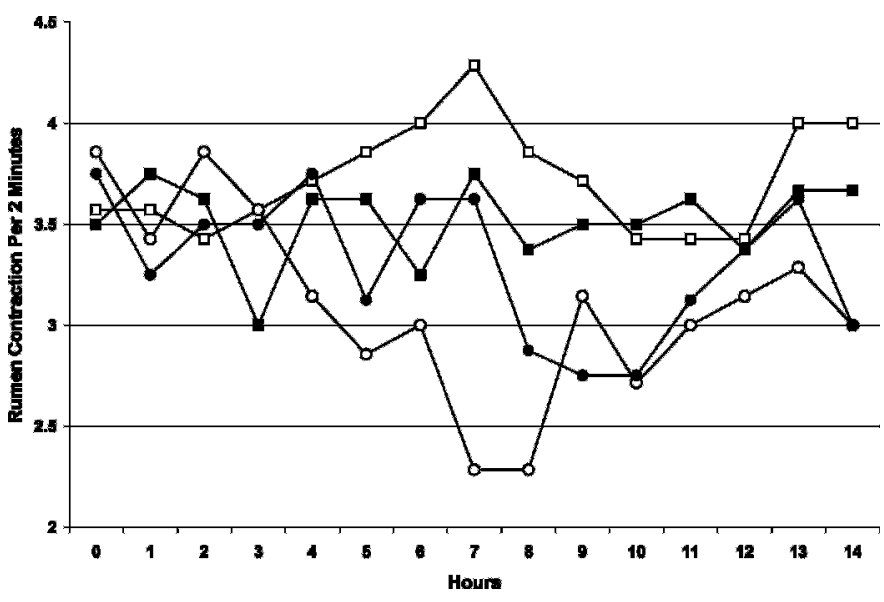

Figure 2. Effects of various treatments on rumen motility. Hour 1 represents baseline measurements. Each value represents the group mean. Legend: $\square$ untreated control; $\square$ drug treatment only; $\bigcirc$ untreated endotoxin-induced mastitis; - endotoxin-induced mastitis and drug treatment.

weights were replaced with an average of the previous and subsequent milk weights. For this herd, the bias introduced by using morning weights to estimate evening weights and vice versa is minimal, as mean morning and evening milking weights for all cows in this study, calculated using only observed weights, varied by less than $1 \%$. One cow from the untreated mastitis group was missing all but one milk weight measurement, and one cow from the same group was missing more than half of the milk weights to be used for analysis. These two cows were removed from statistical analysis of milk production, but their clinical observation data were complete and were used in analysis of treatment effects. Among cows with milk weight data used for statistical analysis, one cow from the control group (no mastitis or drug treatment) was missing 12 of the 24 observations used. Five cows were missing four to six data points, three cows were missing three data points, seven cows were missing two data points, four cows were missing one data point, and eight cows had milk weights for all time points used in the statistical analysis. The replacement of missing milk weights with the average of previous and subsequent weights may reduce the "within-cow" variation in the data, which may increase the likelihood of differences between treatment groups being found to be statistically significant.

\section{RESULTS}

\section{Physiological Variables}

Rumen contractions. (Figure 2) For all groups, baseline observations were not significantly different 


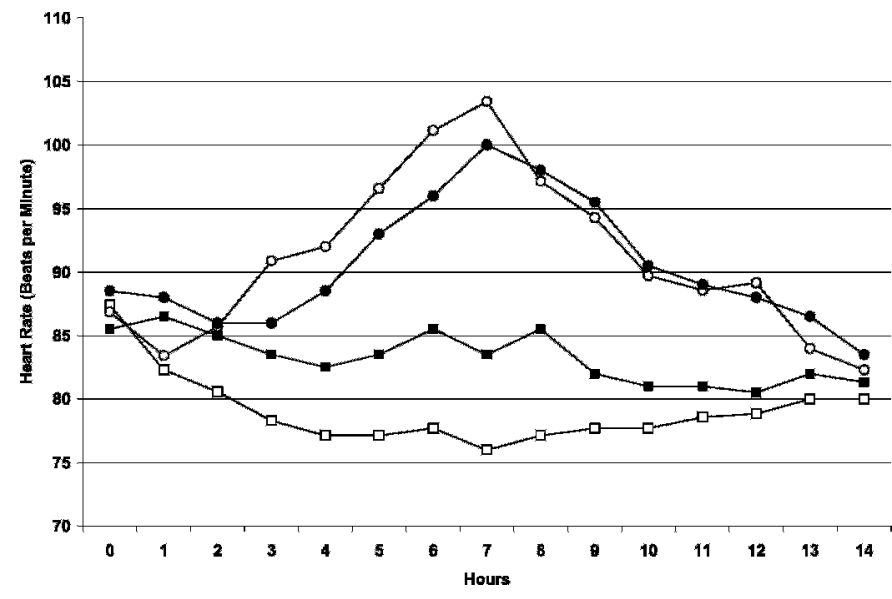

Figure 3. Effects of various treatments on heart rate. Hour 1 represents baseline measurements. Each value represents the group mean. Legend: $\square$ untreated control; $\square$ drug treatment only; $\bigcirc$ untreated endotoxin-induced mastitis; endotoxin-induced mastitis and drug treatment.

$(P=0.8556)$. Both drug treated $(P=0.0413)$ and untreated $(P<0.01)$ cows with mastitis had significantly decreased rumen motility over time compared with the control group. Drug treatment did not significantly affect the magnitude of rumen motility depression in mastitic cows $(P=0.27)$. The group of cows receiving drug treatment alone did not have decreased rumen motility compared with the untreated control group $(P=0.4105)$.

Heart rate. Baseline heart rate for the four groups was not different $(P=0.8611$; Figure 3$)$. Cows receiving only drug treatment had a higher heart rate over time $(P<0.01)$ than untreated controls. Groups with mastitis had the same mean heart rate over time regardless of whether they were treated with isoflupredone acetate or not $(P=0.4524)$. Both mastitic groups had higher mean heart rates than the group receiving only the drug (mastitis plus drug treatment, $(P=0.0245$, mastitis only $(P=0.0112)$ and higher mean heart rates than the untreated control group $(P<0.01$ for both mastitic groups).

Rectal temperature. Mean baseline temperatures for all groups were not significantly different $(P=$ 0.6674 ; Figure 4$)$. Temperature changes over time were the same for all cows with mastitis $(P=0.5307)$ and for all cows without mastitis $(P=0.7663)$, regardless of drug treatment status. Mastitic cows with or without drug treatment had higher mean temperatures over time than did control cows or cows receiving only drug treatment $(P<0.01$ for all groups).

Mammary surface area. Changes in mammary surface area over the $14 \mathrm{~h}$ following the introduction of LPS into the mammary gland were not different for

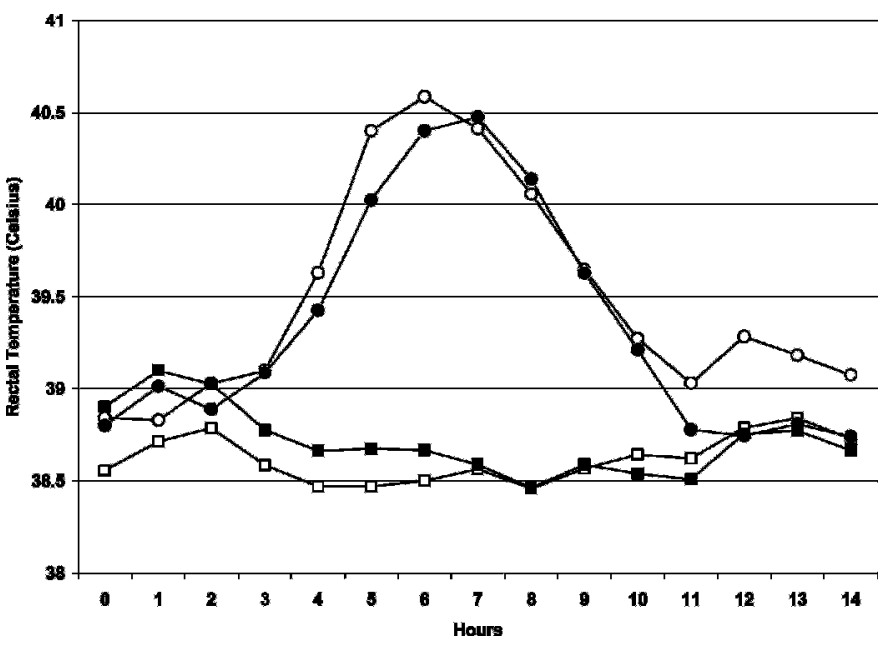

Figure 4. Effects of various treatments on rectal temperature. Hour 1 represents baseline measurements. Each value represents the group mean. Legend: $\square$ untreated control; $\square$ drug treatment only; $\bigcirc$ untreated endotoxin-induced mastitis; mastitis and drug treatment.

drug treated and untreated mastitic cows $(P=0.097$; Figure 5).

Milk production. Mean baseline milk production for the $4 \mathrm{~d}$ preceding the initiation of the trial was not different between treatment groups $(P=0.5544$; Figure 6).

There were no statistically significant differences in milk weights between any groups for the day of endotoxin challenge and drug administration $(P=0.0858)$. On the following day, both treatment groups with mastitis had lower milk production than the control group

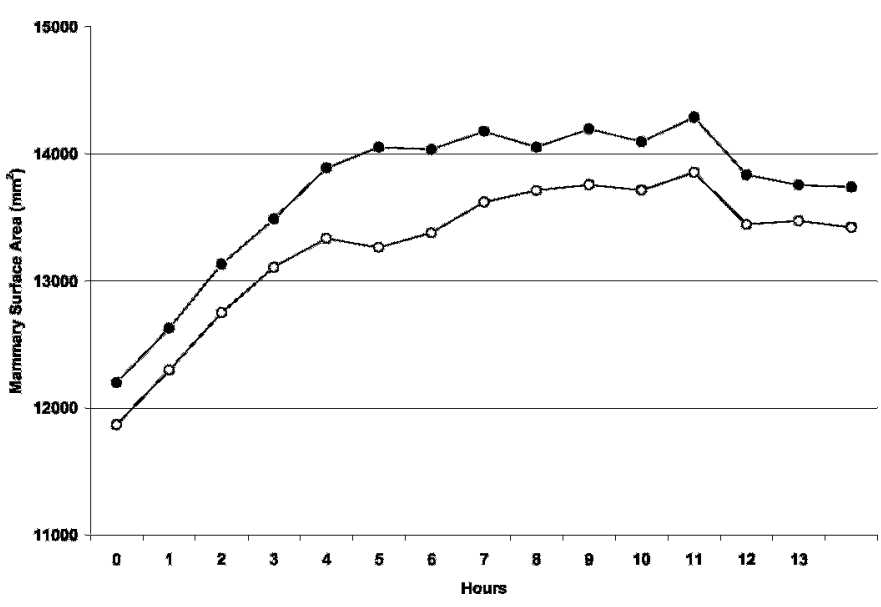

Figure 5. Effects of various treatments on mammary swelling. Hour 1 represents baseline measurements. Each value represents the group mean. Legend: $\square$ untreated control; $\square$ drug treatment only; $\bigcirc$ untreated endotoxin-induced mastitis; $\bullet$ endotoxin-induced mastitis and drug treatment. 


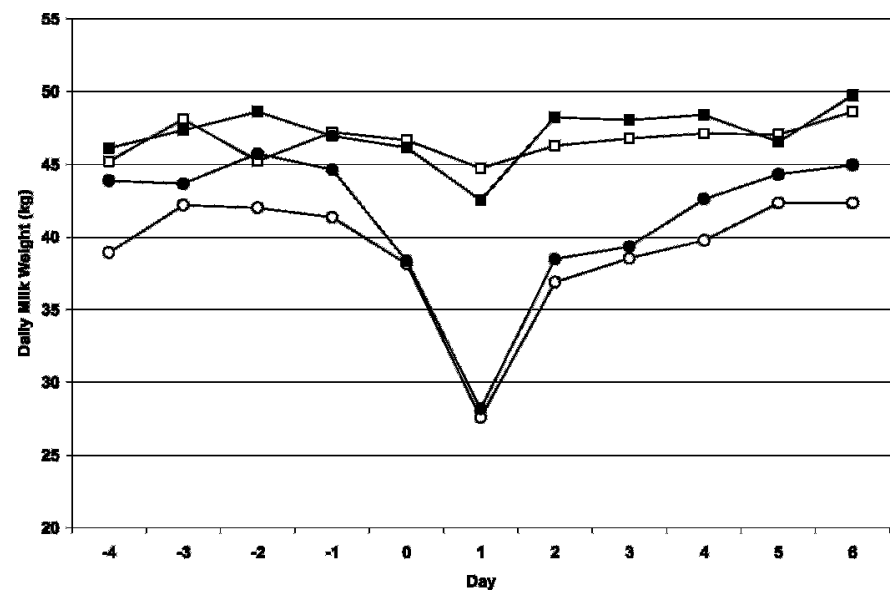

Figure 6. Effects of various treatments on milk production. Treatments were administered on $d 0$. Each value represents the group mean. Legend: $\square$ untreated control; $\square$ drug treatment only; $\bigcirc$ untreated endotoxin-induced mastitis; $\bullet$ endotoxin-induced mastitis and drug treatment.

$(P<0.05)$. By the second day following the induction of mastitis, there were once again no statistically significant differences in milk production between the groups $(P>0.05)$. For the drug-treated group without mastitis, there was no significant difference in milk production compared with the control group at any time point $(P>0.05)$.

\section{DISCUSSION}

Previous work investigating the effect of anti-inflammatory steroid drugs in cows with endotoxic mastitis has largely been focused on the effect of drug administration prior to or concurrent with the induction of mastitis. Carroll et al. (1965) gave 9-alpha fluoroprednisolone acetate (also called isoflupredone acetate) using a variety of regimens, then induced mastitis using Aerobacter aerogenes bacteria introduced into the mammary gland. In that study, doses of 50 and $100 \mathrm{mg}$ of 9-alpha fluoroprednisolone were given once intramuscularly, $250 \mathrm{mg}$ was given twice intramuscularly at a 10-h interval; $200 \mathrm{mg}$ was given three times intramuscularly at 12 -h intervals, or $500 \mathrm{mg}$ was given by the intramammary route into each front quarter once. Single dose regimens were administered concurrently with the intramammary administration of bacteria, while multiple dose regimens had the final dose administered concurrently with the induction of mastitis. Nine-alpha fluoroprednisolone decreased mammary swelling following the induction of mastitis and caused decreased milk production before the induction of mastitis in multiple-dose regimens. Milk production beyond $48 \mathrm{~h}$ after the mastitic episode was not recorded, but might not be relevant to today's high producing dairy cows in any case; the cows enrolled in this study $37 \mathrm{yr}$ ago were producing between 25 and $45 \mathrm{lb}$ of milk per day, much less than typical modern dairy cows.

Administration of $30 \mathrm{mg}$ of dexamethasone intramuscularly to cows concurrently with the induction of mastitis using Escherichia coli bacteria affected physiological parameters and milk production in treated cows (Lohuis et al., 1988). When compared with salinetreated controls, cows receiving dexamethasone had reduced neutropenia, reduced local signs of inflammation, diminished decreases in rumen amplitude, and increased rectal temperature during the acute phase of mastitis. Milk production over the $14 \mathrm{~d}$ following induction of mastitis was markedly increased in cows receiving dexamethasone treatment compared with salinetreated controls. Dexamethasone also decreased the shift in milk production ratio from rear to front quarters in this study in which all cows were infected in a rear quarter. This suggests that the drug directly affected the mastitic gland, rather than facilitating a shift in production to the unaffected glands.

Further work by Lohuis et al. (1989) examined three different steroidal drugs. Flumethasone at a dose of 5 $\mathrm{mg}$ and dexamethasone at a dose of $30 \mathrm{mg}$ were given intramuscularly at the same time as induction of mastitis using purified bacterial endotoxin. Prednisolone was given at a dose of $40 \mathrm{mg}$ by the intramammary route at 0,2 , or $4 \mathrm{~h}$ after the administration of endotoxin. Intramammary administration of prednisolone $2 \mathrm{~h}$ after administration of endotoxin abolished the febrile response and diminished the endotoxin-induced rise in heart rate. Cows given intramuscular steroid drugs in this experiment had less mammary swelling, smaller increase in rectal temperature, smaller increase in heart rate, and less severe neutrophilia and lymphopenia compared with untreated mastitic controls. Milk production effects were not reported.

An endotoxin-induced mastitis model was used to investigate the effect of a very high intravenous dose of $0.44 \mathrm{mg} / \mathrm{kg}$ of dexamethasone; for a typical $500-\mathrm{kg}$ dairy cow this would be $220 \mathrm{mg}$, more than seven times the dose used by Lohuis et al. (Anderson and Hunt, 1989). Dexamethasone was administered two hours after the introduction of endotoxin into the mammary gland. It was stated that there was clinically detectable mammary swelling at the time of drug administration, but not that there were detectable systemic abnormalities at that time. Cows given dexamethasone in this study did not achieve mean rectal temperatures above $39.2^{\circ} \mathrm{C}$, in marked contrast to the increased rectal temperatures observed in cows receiving a much lower dose of dexamethasone in the previously mentioned study. 
The purpose of the study reported here was to investigate whether a moderate dose of the steroidal drug isoflupredone acetate could ameliorate milk production losses and clinical signs when used as it might be used in a clinical setting - at the approved label dose-in a cow with systemic signs of illness. Isoflupredone acetate does not have a labeled indication for intravenous administration, but such use is not prohibited. The results obtained when the drug was given after the development of clinical signs were not entirely consistent with the results of the earlier studies, which generally administered steroid drugs before the development of clinical signs. Unlike previous studies, inhibition of rumen motility (measured in this study using the crude method of auscultation) and increased mammary gland surface area were not alleviated. Isoflupredone acetate also did not affect febrile changes in rectal temperature in treated cows with mastitis; it neither increased the magnitude of change as a moderate dose of dexamethasone had done previously, nor decreased the magnitude of change as a large dose of dexamethasone had done. Isoflupredone did not decrease heart rate in treated cows with mastitis as compared with untreated cows with mastitis. An unanticipated finding in this study was that cows receiving drug treatment only, without mastitis, had a higher heart rate over the observation period than did untreated controls. This may be partly or entirely due to stress, as the cows receiving drug treatment had jugular venipuncture performed eight times during the 14-h observation period for a concurrent pharmacokinetic study.

Isoflupredone acetate did not affect milk production in mastitic or healthy cows in this study. Mastitic cows with or without drug treatment had significantly decreased milk production for $1 \mathrm{~d}$ after the induction of mastitis. This drop in mean production was approximately $30 \%$ of baseline for both groups on the first day after the trial, and it had rebounded to approximately a $10 \%$ loss for both groups by the next day. This is a considerably milder effect than was achieved with live bacterial model used by Lohuis et al. in 1988. In that study, the induction of mastitis using live Escherichia coli bacteria resulted in milk production being 48 to $100 \%$ reduced in untreated cattle $14 \mathrm{~d}$ after the initiation of mastitis. Because the model used in the current study caused only mild and transient milk production loss, drug treatment effects would have to be very strong or study power (cow numbers) would have to be quite high to detect an effect of drug treatment on milk production. Isoflupredone acetate has less than half the anti-inflammatory potency of dexamethasone (Langston, 1993). The unfortunate loss of all data for two cows due to lameness and milk production data for two cows due to computer malfunction reduced the power of this study to detect any significant treatment effects.

In conclusion, the data from this study suggest that the steroidal drug isoflupredone acetate, when administered at the label dose after the onset of clinical signs in an endotoxin-induced mastitis model, does not affect the course of clinical signs in the acute phase of mastitis or subsequent milk production.

\section{ACKNOWLEDGMENTS}

This research was funded by Pharmacia Animal Health. The authors wish to thank the management and staff of the Ankeny ISU Dairy, Doug Ensley, Ron Erskine, Rich Evans, and Zach Frederick.

\section{REFERENCES}

Anderson, K. L., A. R. Smith, R. D. Shanks, L. E. Davis, and B. K. Gustafssson. 1986. Efficacy of flunixin meglumine for the treatment of endotoxin-induced bovine mastitis. Am. J. Vet. Res. 47:1366-1372.

Anderson, K. L., and E. Hunt. 1989. Anti-inflammatory therapy in acute endotoxin-induced bovine mastitis. Vet. Res. Commun. 13:17-26.

Bywater, R. J., K. Godinho, and J. F. Buswell. 1988. Effects of prednisolone on experimentally induced mastitis treated with amoxicillin and clavulanic acid. Proc. 15th World Buiatrics Congress, Palma de Mallorca, Spain.

Carroll, E. J., O. W. Schalm, and J. Lasmanis. 1965. The use of a synthetic corticoid on experimental coliform (Aerobacter aerogenes) mastitis in cattle: The effects of intrammary and intramuscular administration ion the inflammatory response. Am. J. Vet. Res. 26:858-864.

Langston, V. C. 1993. Therapeutic management of inflammation. Pages 8-12 in Current Veterinary Therapy 3. J. L. Howard, ed. W. B. Saunders Company, Philadelphia, PA.

Lohuis, J. A. C. M., W. Van Leeuwen, J. H. Verheijden, A. S. Miert, and A. Brand. 1988. Effect of dexamethasone on experimental Escherichia coli mastitis in the cow. J. Dairy Sci. 71:2782-2789.

Lohuis. J. A. C. M., W. Van Leeuwen, J. H. Verheijden, A. Brand, and A. S. Miert. 1989. Effect of steroidal anti-inflammatory drugs on Escherichia coli endotoxin-induced mastitis in the cow. J. Dairy Sci. 72:241-249

Nickerson, S. C., M. J. Paape, R. J. Harmon, and G. Ziv. 1986. Mammary leukocyte response to drug therapy. J. Dairy. Sci. 69:1733-1742. 